\title{
MEGADOCK-Web: an integrated database of high-throughput structure-based protein-protein interaction predictions
}

\author{
Takanori Hayashi ${ }^{1,2}$, Yuri Matsuzaki ${ }^{2}$, Keisuke Yanagisawa ${ }^{1,2}$, Masahito Ohue ${ }^{1,3,4^{*}}$ and Yutaka Akiyama ${ }^{1,2,3,4,5^{*}}$
}

From The Sixteenth Asia Pacific Bioinformatics Conference

Yokohama, Japan. 15-17 January 2018

\begin{abstract}
Background: Protein-protein interactions (PPIs) play several roles in living cells, and computational PPI prediction is a major focus of many researchers. The three-dimensional (3D) structure and binding surface are important for the design of PPI inhibitors. Therefore, rigid body protein-protein docking calculations for two protein structures are expected to allow elucidation of PPIs different from known complexes in terms of 3D structures because known PPI information is not explicitly required. We have developed rapid PPI prediction software based on protein-protein docking, called MEGADOCK. In order to fully utilize the benefits of computational PPI predictions, it is necessary to construct a comprehensive database to gather prediction results and their predicted 3D complex structures and to make them easily accessible. Although several databases exist that provide predicted PPIs, the previous databases do not contain a sufficient number of entries for the purpose of discovering novel PPIs.
\end{abstract}

Results: In this study, we constructed an integrated database of MEGADOCK PPI predictions, named MEGADOCK-Web. MEGADOCK-Web provides more than 10 times the number of PPI predictions than previous databases and enables users to conduct PPI predictions that cannot be found in conventional PPI prediction databases. In MEGADOCK-Web, there are 7528 protein chains and 28,331,628 predicted PPIs from all possible combinations of those proteins. Each protein structure is annotated with PDB ID, chain ID, UniProt AC, related KEGG pathway IDs, and known PPI pairs. Additionally, MEGADOCK-Web provides four powerful functions: 1) searching precalculated PPI predictions, 2) providing annotations for each predicted protein pair with an experimentally known PPI, 3) visualizing candidates that may interact with the query protein on biochemical pathways, and 4) visualizing predicted complex structures through a 3D molecular viewer.

Conclusion: MEGADOCK-Web provides a huge amount of comprehensive PPI predictions based on docking calculations with biochemical pathways and enables users to easily and quickly assess PPI feasibilities by archiving PPI predictions. MEGADOCK-Web also promotes the discovery of new PPIs and protein functions and is freely available for use at http://www.bi.cs.titech.ac.jp/megadock-web/.

Keywords: Protein-protein interaction, Protein-protein docking, Predicted protein-protein interaction database, MEGADOCK, MEGADOCK-Web

\footnotetext{
*Correspondence: ohue@c.titech.ac.jp; akiyama@c.titech.ac.jp

${ }^{1}$ Department of Computer Science, School of Computing, Tokyo Institute of

Technology, 2-12-1 W8-76 Ookayama, Meguro-ku, Tokyo 152-8550, Japan

Full list of author information is available at the end of the article
} 


\section{Background}

Many proteins interact with each other in nature [1]; these interactions are called protein-protein interactions (PPIs). PPIs play crucial roles in living cells, including signal transduction and regulation of metabolic pathways. In recent years, PPI inhibitors and candidate compounds have also been developed [2, 3] in drug discovery to treat PPI-related diseases. Therefore, understanding of PPIs is important not only for elucidation of biological phenomena but also for pharmaceutical research. PPIs are identified by biochemical experiments, such as the yeast two-hybrid method [4], mass spectrometry [5], and cocrystallization of protein complexes [6]. Although many proteins and their functions have been discovered each year, the discovery of PPIs by biochemical assays is time-consuming and expensive. In addition, several PPIs are involved in protein signaling networks, and many attempts have been made to elucidate the exhaustive interactions among groups of proteins within a specific cellular function category through experiments and calculations [7-9]. In order to improve our understanding of biological processes, computational prediction of PPIs, which is less time-consuming and less expensive than experimental approaches, is needed to discover proteins with a high likelihood of involvement in PPIs $[10,11]$.

Computational PPI prediction methods can be categorized into three types: 1 ) those utilizing the amino acid sequence information of the protein [12-14], 2) those based on co-evolution information [15], and 3) those using the three-dimensional (3D) structure of the protein [16-22]. The third method, based on the protein 3D structure, has been shown to be promising because elucidation of the 3D structure and binding surface localization of the protein can facilitate the design of PPI inhibitors [23, 24]. Methods using the 3D structure of proteins are further divided into two types: 3 -a) methods based on sequence homology and structural similarity with known complex structures [16-18], and 3-b) methods based on rigid body docking of a pair of protein structures [19-22]. Since rigid body docking for two protein structures does not require a priori PPI information, PPIs whose interfaces are not necessarily similar to known complexes in terms of 3D structures or of amino acid compositions may be found by rigid body docking. To predict such novel PPIs, we developed a structure-based PPI prediction software program named MEGADOCK [25]. MEGADOCK uses a template-free (i.e., no reference to experimentally determined cocrystal structures) and fast Fourier transform (FFT)-based rigid body protein-protein docking method. Since the evaluation function of MEGADOCK is computable at high speed, MEGADOCK is faster than other template-free docking tools; for example, MEGADOCK is $\sim 10$ times faster than ZDOCK $[25,26]$. Additionally, its implementation of multithreading, GPU acceleration, and MPI parallelization enables 30,000 PPI predictions in $2 \mathrm{~h}$ with 70 -node GPU-equipped computing clusters [27].

However, in order to utilize PPI predictions (including predicted 3D complex structures), it is necessary to archive prediction results and their $3 \mathrm{D}$ structures and make this information easily accessible. For example, some existing databases, such as PrePPI [16], HOMCOS [17], PRISM [28], and Interactome3D [29], provide PPI predictions. PrePPI provides highly confident PPI predictions by combining structural, functional, evolutionary, and expression information. HOMCOS can predict PPIs on demand using query protein homology. PRISM can predict PPIs on demand based on known template protein-protein interfaces and provides a small database that includes PPI predictions. Interactome3D provides PPI predictions within PPI networks. Unfortunately, it is difficult for such systems to identify PPIs having 3D structures that differ from those of known complexes because they are based on a known PPI template. For example, it is difficult to search the predicted complex structures of adenomatous polyposis coli (APC) protein and Axin protein, a well-known PPI pair [30, 31], using these databases. In the PrePPI database, no predicted structures of the APC and Axin complex are available because PrePPI shows only high-prediction-scored complex structures. In the case of HOMCOS, it is impossible to predict the PPI because homologous proteins consisting of heterodimers have not been reported. In PRISM, the PPI prediction between AOC and Axin is not provided because of the small size of the database. Although PRISM can build predicted complex structure models on-demand, it takes about $1 \mathrm{~h}$ to obtain the model. In the case of Interactome3D, a predicted complex structure model is not obtained because it provides only a part of the experimentally determined structure, even if the full-length structure of APC exists Therefore, a novel database that contains exhaustive PPI predictions from the protein structure is needed to discover PPIs that have structures different from those of known PPIs.

In this study, we developed a database containing comprehensive PPI predictions by MEGADOCK among 7528 representative structures of human protein chains registered in the Protein Data Bank (PDB) [32] (a total of 28,331,628 PPI predictions) and a web interface for the database, named MEGADOCK-Web. MEGADOCK-Web provides the following four powerful functions in order to make the predicted PPI information more useful: 1) searching PPI predictions, 2) providing annotations for each predicted protein pair with known PPIs, 3) visualizing candidates of interactions with query proteins in biochemical pathways, and 4) visualizing predicted complex 
structures using a web browser-based 3D molecular viewer. These functions will allow users to easily access comprehensive PPI predictions from a web browser and facilitate discovery of unknown PPIs.

\section{Construction and contents}

In this section, we describe the construction of MEGADOCK-Web and the contents stored in MEGADOCK-Web. Figure 1 shows the database schema of MEGADOCK-Web. The database of MEGADOCK-Web consists of three models: 1) Protein, 2) Docking, and 3) Pathway. These attributes provide protein structure, predicted PPIs annotated with experimentally known PPI information, and biochemical pathway information in detail.

\section{System architecture}

We developed MEGADOCK-Web using Play Framework [33], which is a Java and Scala's web application framework including components and application programming interfaces (APIs) necessary for web application development. Figure 2 depicts the system architecture of MEGADOCK-Web. MEGADOCK-Web adopts a client-server model in the same way as a general web application. Requests from clients are classified into requests to static resources (such as protein PDB files and docking result files) and requests to the web pages. In the former case, Apache directly returns the corresponding resources to clients. In the latter case,
Apache redirects the requests to the web application running on the local port. For server-side implementation, we stored information in the relational database $\mathrm{H} 2$ Database Engine [34] with MySQL [35] wrapped around Play Framework. Receiving requests from users invokes the web application, which generates HTML dynamically to refer to the database. The server-side implementation is multithreaded to respond immediately. Javascript and jQuery are adopted as client-side implementation tools to control web pages.

\section{Protein structures}

This section explains the protein structures registered in MEGADOCK-Web. Since it is important for supporting drug discovery to integrate data for Homo sapiens, 3775 PDB entries were obtained from the PDB based on the following criteria: protein (not nucleic acids or complexes with nucleic acids); taxonomy of protein is $H$. sapiens; resolution of $2.5 \AA$ or less; and nonredundant so that the sequence similarity is less than $90 \%$ using the PDB search function, which utilizes NCBI BLASTClust [36].

These PDB entries were divided into chain units, and we excluded those with less than 25 residues or with mutations. As a result, 7528 protein chain structures were subjected to PPI prediction. In addition, we annotated each protein chain structure with the ID of UniProt [37] (UniProt AC), the ID of Kyoto Encyclopedia of Genes and Genomes (KEGG) [38], organism name

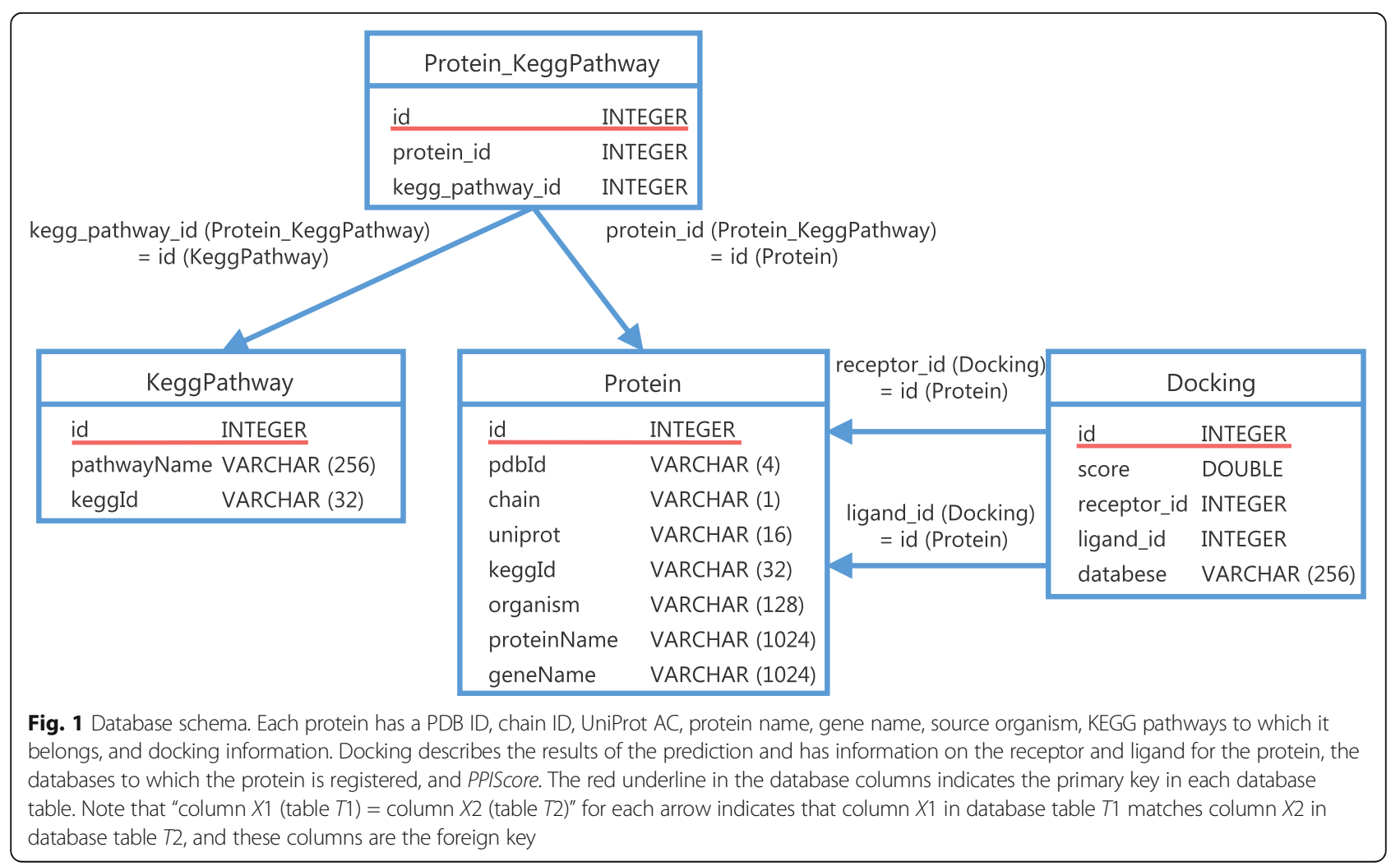




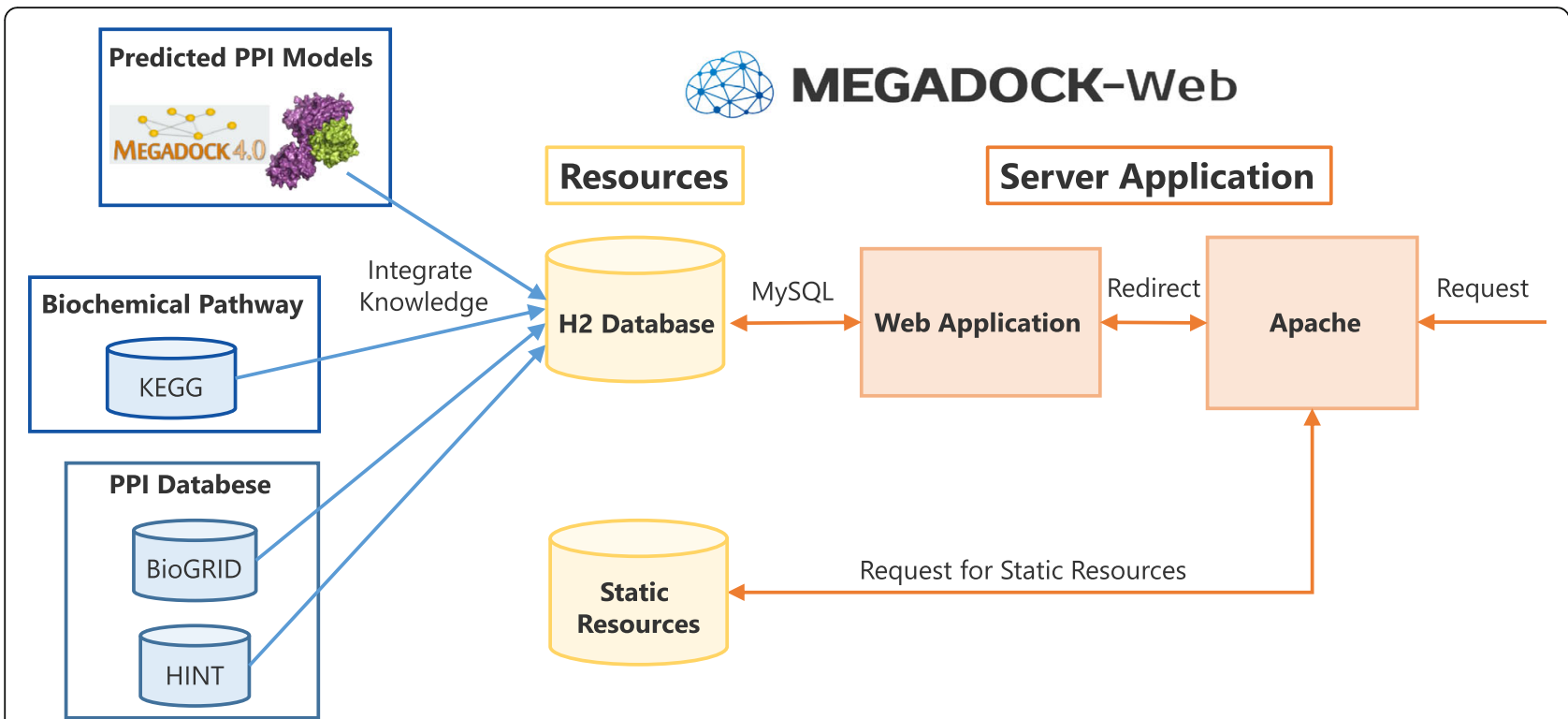

Fig. 2 Archtecture of MEGADOCK-Web. We integrated KEGG, BioGRID, HINT, and predicted PPI information in H2 Database in advance. When a GET or POST request comes, Apache distributes the request depending on whether access to static resources or access to web pages. In the latter case, the web application returns the appropriate page referring to DB with MySQL

(currently only $H$. sapiens), protein name, and gene name using Bioservices [39], a package for Python.

\section{Known PPI pairs}

MEGADOCK-Web shows known PPI pairs as well as vast number of prediction results. HINT [40] (acquired July 13, 2016) and BioGRID [41] (acquired July 13, 2016) are used as databases of known PPI pairs. MEGADOCK-Web shows known PPI information based on UniProt AC because these databases describe known PPIs based on UniProtAC.

\section{Biochemical pathway information}

MEGADOCK-Web uses KEGG pathways as biochemical pathways in order to help understand protein functions. The KEGG pathway is a collection of manually drawn pathway maps representing knowledge on molecular interaction, reaction, and relation networks. We obtained KEGG pathway information for each protein via the web API upon startup of the server just once.

\section{Predicted PPI information}

MEGADOCK-Web integrates exhaustive PPI predictions among 7528 protein chain structures using MEGADOCK. All-to-all PPI predictions (a total of $28,331,628)$ are stored in MEGADOCK-Web.

The procedure for PPI predictions by MEGADOCK is as follows. First, hydrogens and small molecules in HETATM rows in PDB files, such as cofactors and small ligands, were excluded in the predocking process. Secondly, predicted protein-protein conformations were created with MEGADOCK. MEGADOCK outputs the three best translational positions that provide the best value of docking evaluation function (DockingScore) per specific protein rotation, and 3600 patterns of protein rotations are computed, resulting in 10,800 predicted conformations in total. To calculate the DockingScore for each position, we used methods described in our previous papers $[25,42]$. MEGADOCK uses FFT to enable an efficient global docking search on a 3D grid, and calculates shape complementarity, electrostatic interactions, and desolvation free energy [42]. Finally, from each DockingScore, MEGADOCK calculates the PPIScore, which describes the possibility of a PPI. PPIScore is defined as PPIScore $=\left(S_{t o p}-\mu\right) / \sigma$, where $S_{\text {top }}$ is the highest DockingScore in the protein pair, $\mu$ is the average of 10,800 DockingScores, and $\sigma$ is the standard deviation of these scores [24]. Higher PPIScores indicate a higher possibility of a PPI. Although DockingScore cannot be compared between different pairs of proteins, the PPIScore, which is normalized DockingScore, represents how likely the PPI is to occur. The sensitivity of PPI prediction can be adjusted by tuning the threshold value of the PPIScore. Additional file 1: Figure S1 illustrates the precision and recall of MEGADOCK for each threshold. We also reported the ability of MEGADOCK using real pathway datasets in our previous studies $[9,43,44]$.

As a result, we calculated $7528^{2}=56,670,784$ predictions since the number of registered $3 \mathrm{D}$ protein chain structures was 7528 . Reversing the receptor file and the ligand file generally allows predictions to be performed on the same protein pair. Notably, FFT-based docking, 
such as MEGADOCK, usually shows different results (DockingScores) when the order of PDB input pair is switched. In order to avoid such redundancy for users, we stored the protein pairs that had higher PPIScores for prediction between the same PDB files. As a result, MEGADOCK-Web displays ${ }_{7528} \mathbf{C}_{2}+7528$ (homo dimers $)=28,331,628$ PPI predictions. The total computation time was approximately $500 \mathrm{CPU}$ years.

\section{Utility}

Figure 3 provides the page transition diagram of MEGADOCK-Web. In this section, we explain the utilities of MEGADOCK-Web in three situations: 1) searching for PPI candidates of a query protein, 2) searching for PPI candidates on a specific pathway, and 3) assessing the possibility of a PPI for a pair of proteins.

\section{Searching PPI candidates of a query protein}

In this section, we describe the use of MEGADOCK-Web to search PPI candidates of a query protein. First, a user can search with a query word (for example, protein name, PDB ID, UniProt AC, etc.) typed in the "Search Single Protein" window in the Top page (Additional file 1: Figure S2), resulting in transition to the protein selection page (Additional file 1: Figure S3) showing the list of search results. In this page, protein structure hits with the query are listed accompanying identifiable protein information for each protein (PDB ID, chain, UniProt AC, protein name, and gene name). Clicking the "View" button takes users to the PPI prediction list page (Additional file 1: Figure S4). In the PPI prediction list page, proteins are shown in descending order of PPIScore and are paged per 10 items. The list contains information on the following three points for all the proteins in the database: 1) identification information, 2) PPIScores with the query protein, and 3) known PPIs for the query protein. Each row also has a "View" button to transit to the predicted complexes page (Additional file 1: Figure S5) between the query protein structure and the corresponding structure within the row.

On the predicted complexes page, 10 predicted complexes based on the results of the MEGADOCK dockings between the two proteins are displayed with 3D molecule viewers. From the "Download Complex in this page" link, 10 predicted complex structures can be downloaded as PDB files together in zip format. The table includes the following four columns: 1) rank (up to 1000) of the DockingScore, 2) DockingScore, 3) a molecule viewer displaying the 3D structure of the predicted complex, and 4) a PDB file download link for the complex structure. Molmil [45] is adopted as a molecular viewer because of its lightness and stability; the program enables users to rotate and enlarge the structure easily. It is also possible to download the docking results as a text file describing the translation distance, rotation angle of the ligand protein, and DockingScore (ZDOCK 3.0.1 and MEGADOCK output style).

\section{Searching PPI candidates on a specific pathway}

Assuming that a user has a protein of interest known to affect a certain biochemical pathway with unknown interactions, this section describes how to discover PPI candidates in a specific pathway.

First, as in the previous section, the user can search a query by typing in the "Search Single Protein" window at the top of the page (Additional file 1: Figure S2) and select the protein structure in the protein selection page (Additional file 1: Figure S3). Next, in the PPI prediction list page (Additional file 1: Figure S4), the user can move to the pathway selection page (Additional file 1: Figure S6) by clicking the "Show Pathway" button. The pathway selection page displays a list of pathways containing at least one predicted binder. The predicted binder is defined as a protein with a PPIScore of greater than or equal to the user-defined threshold.

The threshold of PPIScore can vary from 6.0 to 12.0 with steps of 1.0 (default value is 8.0). Lowering the threshold increases the sensitivity and decreases the specificity. In the pathway selection page, links to the KEGG pathways to which the predicted binders belong are displayed. Note that the query protein does not always exist on the KEGG pathway; obtaining many predicted binders on the pathway without the query protein indicates a possibility of unknown interactions of the query

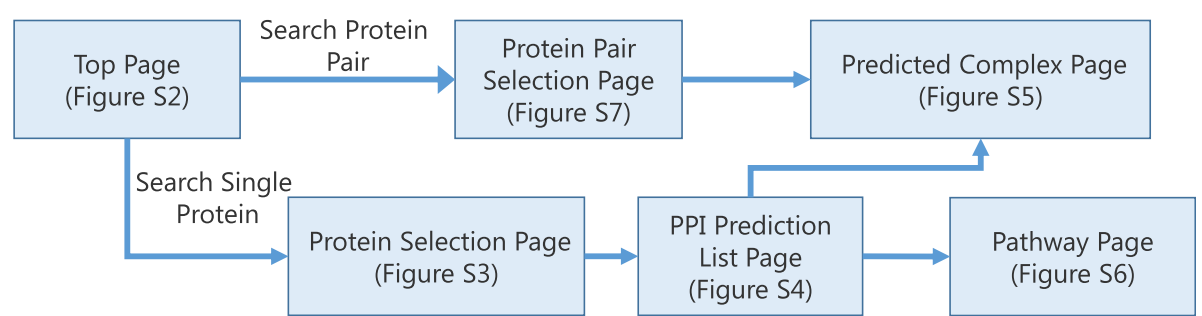

Fig. 3 Page transition diagram of MEGADOCK-Web. For a single query, users can transit to the PPI prediction list page via the protein selection page. From this page, it is possible to transit to the pathway selection page to which the predicted binder belongs and to the prediction complex display page. For two queries, it is possible to transit to the prediction complex display page through the protein pair selection page 
protein in the pathway. Furthermore, in order to display only pathways with specific functions, it is possible to filter the pathways by inputting a word or phrase in the "Pathway Filtering" window at the top of the pathway selection page. Clicking the link takes the user to the corresponding KEGG page, in which the query protein is colored in blue, and predicted binders are in red.

\section{Assessing the possibility of PPIs for a pair of proteins}

MEGADOCK-Web provides a functionality to assess the possibility of interactions with two proteins from functional and structural points of view. First, the user can search for the two proteins in the "Search Protein Pairs" window at the top of the page, resulting in transition to the protein pair selection page (Additional file 1: Figure S7). Selection of protein structures for each query is shown at the upper part of the page, whereas PPIScores of all combinations of pairs of those protein structures are shown in descending order in the lower part of the page. The upper table has information similar to that of the protein selection page. If the structures of proteins to be used have already been specified, the predicted complexes page (Additional file 1: Figure S5) can be accessed by choosing the individual structure of each protein in the upper table.

The lower part of the page is useful if the structures of proteins to be used have not yet been determined. Since pairs of protein structures are presented in descending order of PPIScores, higher PPIScore structures (structures with higher PPI possibility) can be found easily. From the "View" link, it is also possible to transit to the predicted complex page (Additional file 1: Figure S5) of the corresponding proteins.

\section{Discussion}

\section{How to utilize MEGADOCK-Web: Concrete examples}

In this section, three case studies with specific proteins are explained to provide examples of the use of MEGADOCK-Web.

\section{Case 1: APC and Axin (recalling a known PPI)}

Cancer is one of the most serious diseases in humans. APC is a cancer-related protein; thus, PPI candidates with APC are likely to be related to cancer. In this section, we describe a search for PPI candidates of APC using MEGADOCK-Web.

First, when APC is specified as a query, structure information of APC is displayed as search results. After selecting the structure of 1DEB_A (chain A of 1DEB) from several structures, users are taken to the PPI prediction list page (Fig. 4a). On this page, the pathway selection page with the threshold set to 8.0 is shown in order to view the KEGG pathways to which the predicted binders belong. Pathway filtering can be used to find cancer-related pathways. After filtering with the word "cancer", the user can select breast cancer (KEGG ID: hsa05224). In the identified pathway, Axin (colored in red as a predicted binder) is present next to APC (colored in blue as the query protein; Fig. 4b). In order to more deeply assess the possibility of an interaction between APC and Axin, the user can use the pair query function and show the PPI prediction in detail. When searching APC and Axin as a pair query, some structures are found for both proteins. Selecting 1DEB_A and 1DK8_A results in transition to the predicted complex page (Fig. 4c). In the predicted complex page, predicted complexes are browsed, and the possible interactions can be assessed.

By following the above procedure, MEGADOCK-Web can facilitate the assessment of a specific PPI. In fact, the interaction between APC and Axin has been revealed experimentally [31].

\section{Case 2: Sons of Sevenless (SOS) and SH2B adaptor protein 2 (SH2B2) (exploring a novel PPI)}

The potential for identification of PPIs was described in the previous section. Here, we demonstrate the ability to identify a feasible new PPI using MEGADOCK-Web. We explain how to search unknown PPIs against SOS protein, which is also associated with cancer, as an example.

First, the pathway selection page is chosen, as described in the previous section, and the list includes the neurotrophin signaling pathway (hsa04722). In this pathway, an adaptor protein containing Pleckstrin homology (PH) and Src homology-2 (SH2) domains (APS), also known as SH2B2, is adjacent to SOS (Fig. 5a). Second, in order to search the possibility of an interaction between these proteins, searching SOS and $\mathrm{SH} 2 \mathrm{~B} 2$ as a pair query brings the user to the protein pair selection page, after which the user selects the predicted complex of 1DBH_A and 1Q2H_B with the highest score in the table (Fig. 5b). On the predicted complexes page (Fig. $5 \mathrm{c}$ ), the possibility of an interaction is assessed. The PPIScore between 1DBH_A and 1Q2H_B was 8.2, indicating a possibility of a PPI.

The PPI database STRING DB stores experimental information and literature-based predictions [46]. Analysis using the database also supported the possibility of an interaction between SOS and SH2B2 when searching their UniProt ACs Q07889 and O14492, respectively. These results suggested that it may be useful to further investigate the interaction between $\mathrm{SOS}$ and $\mathrm{SH} 2 \mathrm{~B} 2$ (APS). 


\section{a}

Protein Information

\begin{tabular}{|c|c|} 
Your Query: 1deb & \\
\hline Protein Names & Adenomatous polyposis coli protein \\
\hline Gene Names & APC DP2.5 \\
\hline PDB ID & 1DEB (Chain A) \\
UniProt AC & P25054 \\
\hline
\end{tabular}

\section{Predicted PPI Information}

Show KEGG pathways in candidates

PPIScore > 8 . Show Paliway

\begin{tabular}{|c|c|c|c|c|c|c|c|}
\hline $\begin{array}{l}\text { UniProt } \\
\text { AC }\end{array}$ & Organism & Protein Names & Gene Names & PDB ID & $\begin{array}{l}\text { PPI } \\
\text { Score }\end{array}$ & View & Databases \\
\hline P25054 & $\begin{array}{l}\text { Homo } \\
\text { sapiens }\end{array}$ & $\begin{array}{c}\text { Adenomatous } \\
\text { polyposis coli protein }\end{array}$ & APC DP 2.5 & $\begin{array}{c}\text { 1DEB } \\
\text { (Chain B) }\end{array}$ & 13.4563 & View & 测 圆 \\
\hline Q8N4E7 & $\begin{array}{l}\text { Homo } \\
\text { sapiens }\end{array}$ & Ferritin; mitochondrial & FTMT & $\begin{array}{c}1 \mathrm{R} 03 \\
\text { (Chain A) }\end{array}$ & 12.2521 & View & \\
\hline Q09013 & $\begin{array}{l}\text { Homo } \\
\text { sapiens }\end{array}$ & $\begin{array}{l}\text { Myotonin-protein } \\
\text { kinase }\end{array}$ & $\begin{array}{l}\text { DMPK } \\
\text { DM1PK } \\
\text { MDPK }\end{array}$ & $\begin{array}{c}\text { 1WT6 } \\
\text { (Chain D) }\end{array}$ & 11.9903 & View & \\
\hline P08670 & $\begin{array}{l}\text { Homo } \\
\text { canione }\end{array}$ & Vimentin & VIM & $\begin{array}{c}1 \mathrm{GK} 4 \\
\text { (I hain RI }\end{array}$ & 11.5430 & View & \\
\hline
\end{tabular}

b

Scaffold

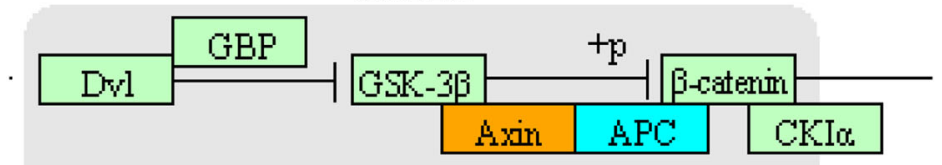

c

Docking Information

\begin{tabular}{|l|c|c|c|}
\hline \multicolumn{2}{|c|}{ Protein Names } & \multicolumn{2}{c|}{ Gene Names } \\
\hline Protein 1 & Axin-1 & AXIN1 AXIN & 1DK8 (Chain A) \\
\hline Protein2 & Adenomatous polyposis coli protein & APC DP2.5 & 1DEB (Chain A) \\
\hline
\end{tabular}

Docking results from rank 1 to 10

Download complex in this page

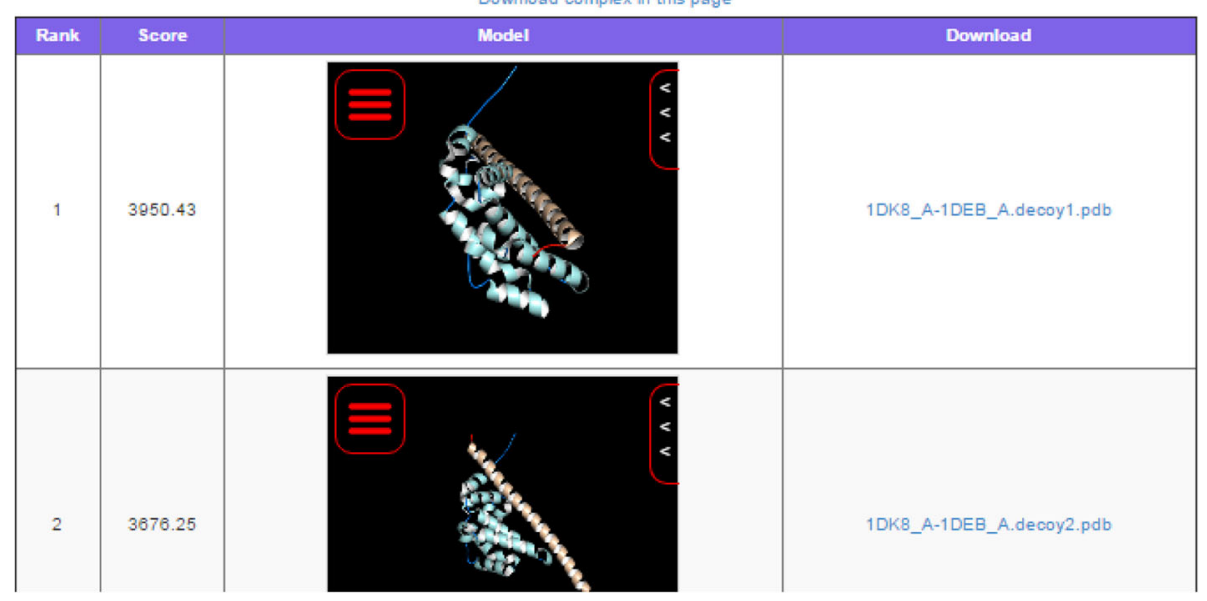

Fig. 4 An example of page transition when searching for protein interaction candidates and APC. a The PPI prediction list page of APC. b Part of the KEGG pathway (breast cancer, hsa05224). The query protein (APC) is colored in blue, and the predicted binders (Axin) are in red. c The predicted complex page of 1DEB_A (APC) and 1DK8_A (Axin) 


\section{a hormotherodirues}

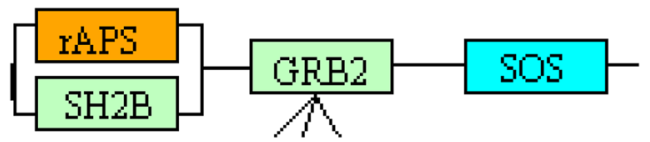

b

PPI Score Information

\begin{tabular}{|c|c|c|l|}
\hline Protein1 (PDBID_Chain) & Protein2 (PDBID_Chain) & PPI Score \\
\hline 1DBH_A & 1Q2H_B & 8.2081 & View \\
\hline 1Q2H_A & 1DBH_A & 7.5399 & View \\
\hline 1Q2H_C & 1DBH_A & 6.8078 & View \\
\hline
\end{tabular}

C

Docking Information

PPIScore: 8.2081 Show KEGG Pathway

\begin{tabular}{|c|c|c|c|}
\hline & Protein Names & Gene Names & PDB ID \\
\hline Protein 1 & Son of sevenless homolog 1 & sos1 & $1 \mathrm{DBH}($ Chain A) \\
\hline Protein2 & $\mathrm{SH} 2 \mathrm{~B}$ adapter protein 2 & $\mathrm{SH} 2 \mathrm{~B} 2 \mathrm{APS}$ & $1 \mathrm{Q} 2 \mathrm{H}($ Chain $\mathrm{B})$ \\
\hline
\end{tabular}

Docking results from rank 1 to 10

Download complex in this page

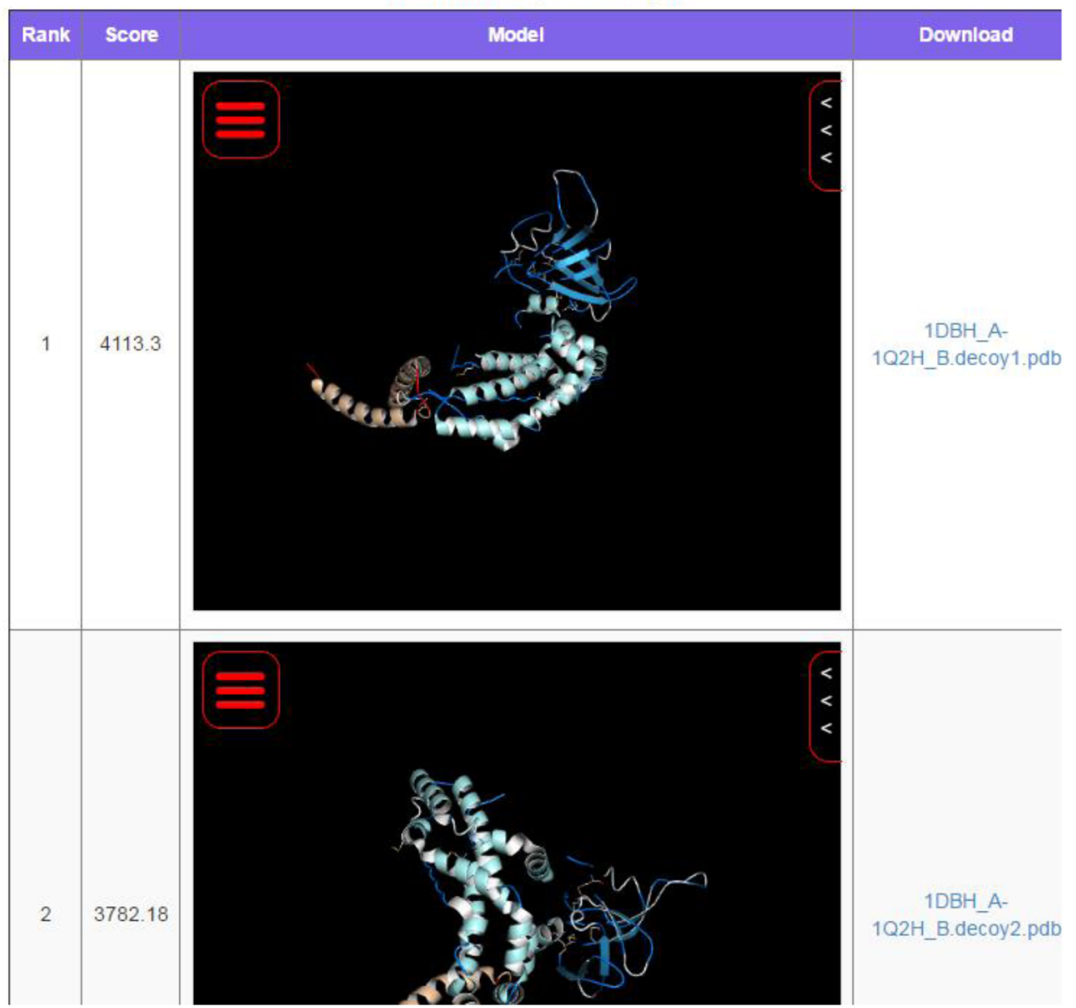

Fig. 5 An example of page transition when searching for SOS protein interaction candidates. a Part of the KEGG pathway (neurotrophin signaling pathway, hsa04722). The query protein (SOS) is colored in blue, and the predicted binder (APS) is in red. Note that the notation of rAPS (rat ortholog of human APS) is considered to be a mistake in APS of KEGG. $\mathbf{b}$ The table in the lower part of the protein pair selection page for SOS and SH2B2 (APS). c The predicted complex page for 1DBH_A (SOS) and 1Q2H_B (SH2B2) 
Case 3: Membrane Metallo-endopeptidase (MME) and methylenetetrahydrofolate dehydrogenase 1 (MTHFD1) (predicting the complex structure of a known PPI)

MME and MTHFD1 are a pair of proteins that interact in prostate cancer cells [47]; however, the complex structure of these proteins has not been determined. MEGADOCK-Web can provide predicted complex structures for these proteins.

When MME and MTHFD1 are searched as queries, candidate structures are obtained. Here, $1 \mathrm{R} 1 \mathrm{H}$ [48] describes the complex structure of MME and an inhibitor, and 1A4I [49] describes a homodimer complex structure of MTHFD1. Thus, the user may select 1R1H_A and 1A4I_B, and the predicted complexes are displayed in the predicted complex page. Figure $6 \mathrm{a}$ shows the predicted complex with Rank 1, and Fig. 6b shows that with Rank 2. The predicted complexes are created for different interaction surfaces. Therefore, users are able to discuss the possibility of PPIs on various interaction surface candidates.

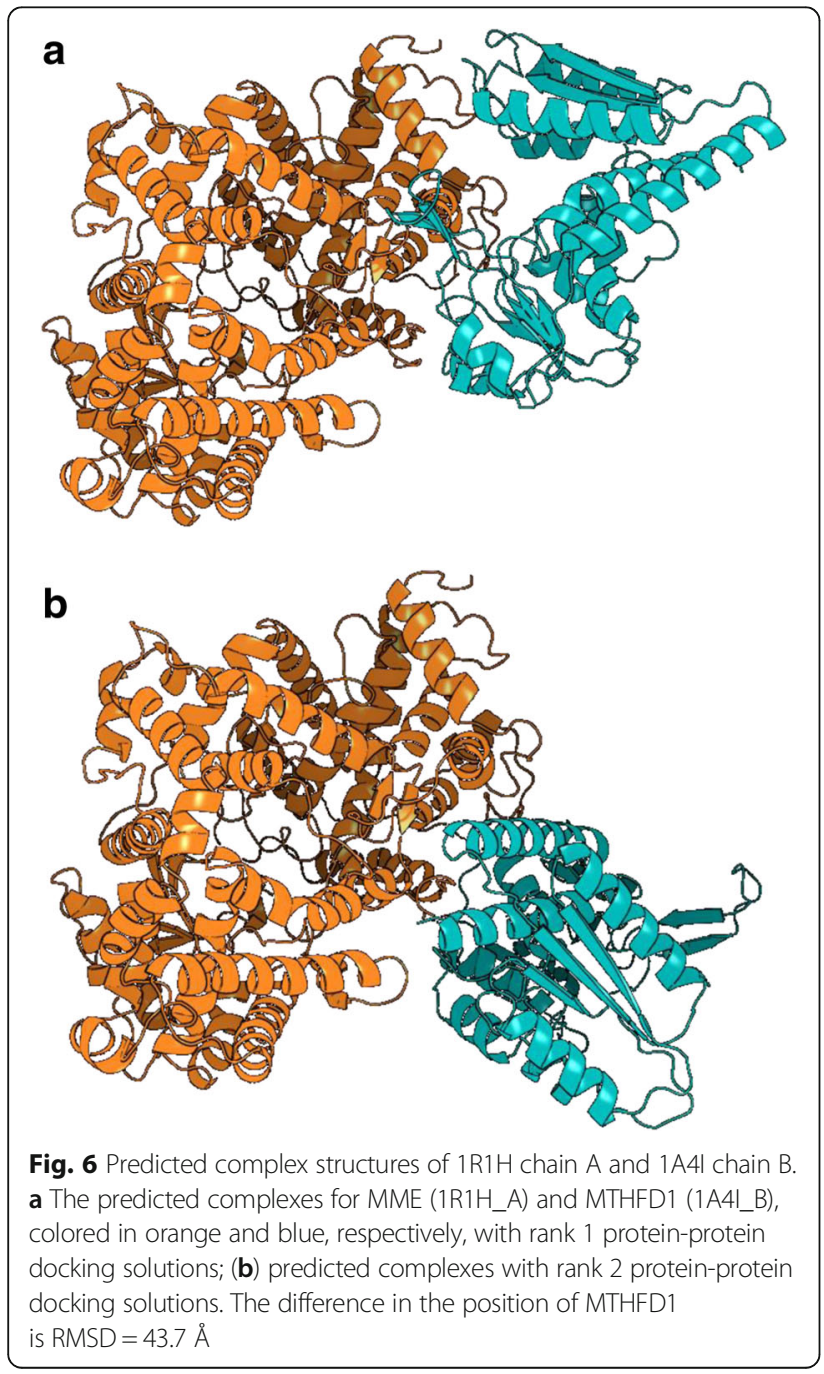

Comparison with other databases

Some existing databases contain 3D structural information similar to MEGADOCK-Web, such as PrePPI [16], HOMCOS [17], PRISM [28], and Interactome3D [29]. Table 1 shows a comparison of these databases and MEGADOCK-Web. MEGADOCK-Web was found to be superior in the following points: 1) provides many PPI predictions, 2) allows the user to search for pair proteins, 3) has a shorter response time, 4) permits viewing of some prediction complexes, and 5) allows mapping of proteins onto biochemical pathways.

First, MEGADOCK-Web contains a larger number of PPI predictions than other databases because it uses MEGADOCK, a rapid rigid docking tool. For example, MEGADOCK-Web provides 10 times more PPI predictions than PrePPI and 100 times more PPI predictions than Interactome3D. Second, MEGADOCK-Web supports both single protein and paired protein queries. Thus, it is possible to conduct searches from various perspectives. Furthermore, since PrePPI displays only one prediction model, users cannot discuss the predicted complexes on various interaction surfaces. In contrast, MEGADOCK-Web enables users to investigate the complexes because up to 1000 candidate complexes can be shown in the predicted complexes page. Finally, since proteins are mapped on the biochemical pathways (KEGG pathways), MEGADOCK-Web helps users to discuss the possibility of PPIs based on the biological roles of the predicted interactions.

\section{Future plans}

MEGADOCK-Web is expected to help to annotate proteins with unknown functions by providing comprehensive PPI predictions, irrespective of species. This program can be expected to help researchers discover new PPIs more broadly if we expand the database to include other species (e.g., Saccharomyces cerevisiae, Escherichia coli and Mus musculus). Although the current version of MEGADOCK-Web provides only human proteins, we will expand MEGADOCK-Web to include other species in the near future.

MEGADOCK-Web provides PPI predictions for proteins registered in the database. It will be useful to expand this system to perform docking calculations on-demand and allow browsing of PPI prediction results. With ordinary computers and implementations, long computation times are required for on-demand rigid body docking calculations; however, our system operates at multiple GPU computing nodes at high speeds. In addition, we have already completed a porting of MEGADOCK onto a public cloud computing resource (Microsoft Azure) that can use computing resources according to demand (Ohue $\mathrm{M}$, et al: 
Table 1 Comparison with other PPI prediction databases

\begin{tabular}{|c|c|c|c|c|c|}
\hline & PrePPI [16] & HOMCOS [17] & PRISM [28] & Interactome3D [29] & MEGADOCK-Web \\
\hline Number of PPI predictions & $1,350,000$ & - & 30,475 & 227,815 & $28,331,628$ \\
\hline Comprehensive PPI prediction & & & & & $\checkmark$ \\
\hline Search a single protein query & $\checkmark$ & & $\checkmark$ & $\checkmark$ & $\checkmark$ \\
\hline Search a pair protein query & $\checkmark$ & $\checkmark$ & $\checkmark$ & $\checkmark$ & $\checkmark$ \\
\hline Viewing of multiple modeled complex structures & & $\checkmark$ & $\checkmark$ & $\checkmark$ & $\checkmark$ \\
\hline Mapping biochemical pathways & & & & $\checkmark$ & $\checkmark$ \\
\hline Template-free PPI prediction & & & & & $\checkmark$ \\
\hline
\end{tabular}

The numbers of archived PPI predictions as of June 1, 2017 are shown. Because HOMCOS is an on-demand prediction server, the number of PPI predictions is shown as ' - .' ' $\checkmark$ ' and blank cells indicate enable and disable

MEGADOCK-Azure: cloud-based high-performance protein-protein interaction prediction system on Azure HPC, in preperation). We will release a service for on-demand PPI prediction in the near future.

\section{Conclusions}

In this paper, we described our new database, named MEGADOCK-Web, which is an integrated database of high-throughput structure-based PPI predictions. MEGADOCK-Web is freely available for use at http:// www.bi.cs.titech.ac.jp/megadock-web/.

MEGADOCK-Web provides PPI predictions to users quickly by archiving exhaustive PPI predictions based on docking calculations. MEGADOCK-Web provides the following powerful functions: 1) searching PPI predictions by MEGADOCK, 2) providing annotations for each predicted protein pair with experimentally known PPIs, 3) visualizing candidate interactions with query proteins in biochemical pathways, and 4) visualizing predicted complex structures using a 3D molecular viewer. MEGADOCKWeb also gives comprehensive PPI prediction results in cooperation with biochemical pathways and enables users to easily and rapidly examine PPI possibilities. MEGADOCK-Web is expected to facilitate the discovery of new PPIs and protein functions.

\section{Availability and requirements}

MEGADOCK-Web is freely available for use at http:// www.bi.cs.titech.ac.jp/megadock-web/.

\section{Operating systems}

MEGADOCK-Web is accessed from a web browser; therefore, it is platform-independent.

\section{Browsers}

MEGADOCK-Web has been extensively tested with Google Chrome, Safari, Mozilla Firefox, and Microsoft Edge.

\section{Any restrictions for use}

MEGADOCK-Web is free to use by everyone; there are no restrictions.

\section{Additional files}

Additional file 1: Supplementary Figures. PDF file containing various figures with detailed MEGADOCK performance and MEGADOCK-Web screenshots. (PDF $819 \mathrm{~kb}$ )

\section{Abbreviations}

3D: Three-dimensional; APC: Adenomatous Polyposis Coli; API: Application Programming Interface; APS: an adaptor molecule containing PH and $\mathrm{SH} 2$ domains; FFT: Fast Fourier Transform; GPU: Graphics Processing Unit; HTML: HyperText Markup Language; KEGG: Kyoto Encyclopedia of Genes and Genomes; MME: Membrane Metallo-endopeptidase; MTHFD1: Methylenetetrahydrofolate Dehydrogenase 1; PDB: Protein Data Bank; PPI: Protein-Protein Interaction; SH2: Src homology-2; SH2B2: SH2B adaptor protein 2; SOS: Son of Sevenless

\section{Acknowledgements}

We thank Mr. Kazuki Nagasawa for helping to implement the MEGADOCKWeb database system and Dr. Nobuyuki Uchikoga for testing the MEGADOCK-Web service.

\section{Funding}

This work was partially supported by the Core Research for Evolutional Science and Technology (CREST) "Extreme Big Data" (grant number JPMJCR1303) from the Japan Science and Technology Agency (JST), the Research Complex Program "Wellbeing Research Campus: Creating new values through technological and social innovation" from the Japan Science and Technology Agency (JST), KAKENHI (grant numbers 15K16081, 16K00388, and 17H01814) from the Japan Society for the Promotion of Science (JSPS), Platform Project for Supporting Drug Discovery and Life Science Research (Basis for Supporting Innovative Drug Discovery and Life Science Research) from Japan Agency for Medical Research and Development (AMED), the Regional Innovation and Ecosystem Formation Program "Program to Industrialize an Innovative Middle Molecule Drug Discovery Flow through Fusion of Computational Drug Design and Chemical Synthesis Technology" from the Japanese Ministry of Education, Culture, Sports, Science and Technology (MEXT), Leave a Nest Grant from Leave a Nest, Co., Ltd., and Microsoft Japan, Co., Ltd. The publication charges for this article were funded by JST (CREST JMPJCR1303 and the Research Complex Program).

Availability of data and materials

Not applicable.

\section{About this supplement}

This article has been published as part of BMC Bioinformatics Volume 19 Supplement 4, 2018: Selected articles from the 16th Asia Pacific Bioinformatics Conference (APBC 2018): bioinformatics. The full contents of the supplement 
are available online at https://bmcbioinformatics.biomedcentral.com/articles/ supplements/volume-18-supplement-17.

\section{Authors' contributions}

$\mathrm{TH}, \mathrm{YM}$, and $\mathrm{MO}$ integrated the external resources and participated in the design of the MEGADOCK-Web database and website. MO and YA coordinated the project and provided guidance for system implementations. TH constructed the database and developed the website. TH, YM, and KY designed the use cases of MEGADOCK-Web. TH, KY, MO, and YA wrote the manuscript. All authors read and approved the final manuscript.

\section{Ethics approval and consent to participate}

Not applicable.

\section{Consent for publication}

Not applicable.

\section{Competing interests}

The authors declare that they have no competing interests.

\section{Publisher's Note}

Springer Nature remains neutral with regard to jurisdictional claims in published maps and institutional affiliations.

\section{Author details}

${ }^{1}$ Department of Computer Science, School of Computing, Tokyo Institute of Technology, 2-12-1 W8-76 Ookayama, Meguro-ku, Tokyo 152-8550, Japan. ${ }^{2}$ Education Academy of Computational Life Sciences, Tokyo Institute of Technology, 2-12-1 W8-93 Ookayama, Meguro-ku, Tokyo 152-8550, Japan. ${ }^{3}$ Advanced Computational Drug Discovery Unit (ACDD), Institute of Innovative Research, Tokyo Institute of Technology, 4259 Nagatsutacho, Midori-ku, Yokohama, Kanagawa 226-8501, Japan. ${ }^{4}$ AIST-Tokyo Tech Real World Big-Data Computation Open Innovation Laboratory (RWBC-OIL), National Institute of Advanced Industrial Science and Technology (AIST), 1-1-1 Umezono, Tsukuba, Ibaraki 305-8560, Japan. ${ }^{5}$ Molecular Profiling Research Center for Drug Discovery (molprof), National Institute of Advanced Industrial Science and Technology (AIST), 2-4-7 Aomi, Koto-ku, Tokyo 135-0064, Japan

\section{Published: 8 May 2018}

\section{References}

1. Stelzl U, Worm U, Lalowski M, Haenig C, Brembeck FH, Goehler H, et al. A human protein-protein interaction network: a resource for annotating the proteome. Cell. 2005;122:957-68.

2. Oltersdorf T, Elmore SW, Shoemaker AR, Armstrong RC, Augeri DJ, Belli BA et al. An inhibitor of BCl-2 family proteins induces regression of solid tumours. Nature. 2005:435:677-81.

3. Popowicz GM, Czarna A, Wolf S, Wang K, Wang W, Dömling A, et al Structures of low molecular weight inhibitors bound to MDMX and MDM2 reveal new approaches for p53-MDMX/MDM2 antagonist drug discovery. Cell Cycle. 2010;9:1104-11.

4. Ito T, Chiba T, Ozawa R, Yoshida M, Hattori M, Sakaki Y. A comprehensive two-hybrid analysis to explore the yeast protein interactome. Proc Natl Acad Sci U S A. 2001;98:4569-74.

5. Ho Y, Gruhler A, Heilbut A, Bader GD, Moore L, Adams S, et al. Systematic identification of protein complexes in Saccharomyces Cerevisiae by mass spectrometry. Nature. 2002:415:180-3.

6. Gamble TR, Vajdos FF, Yoo S, Worthylake DK, Houseweart M, Sundquist WI, et al. Crystal structure of human cyclophilin a bound to the amino-terminal domain of HIV-1 capsid. Cell. 1996;87:1285-94.

7. Yachie N, Petsalaki E, Mellor JC, Weile J, Jacob Y, Verby M, et al. Pooledmatrix protein interaction screens using barcode fusion genetics. Mol Syst Biol. 2016;12:863.

8. Stein A, Mosca R, Aloy P. Three-dimensional modeling of protein interactions and complexes is going 'omics. Curr Opin Struct Biol. 2011;21: 200-8.

9. Matsuzaki Y, Matsuzaki Y, Sato T, Akiyama Y. In silico screening of proteinprotein interactions with all-to-all rigid docking and clustering: an application to pathway analysis. J Bioinforma Comput Biol. 2009;7:991-1012.
10. Skrabanek L, Saini HK, Bader GD, Enright AJ. Computational prediction of protein-protein interactions. Mol Biotechnol. 2008;38:1-17.

11. Matsuzaki Y, Uchikoga N, Ohue M, Akiyama Y. Rigid-docking approaches to explore protein-protein interaction space. Adv Biochem Eng Biotech. 2017; 160:33-55.

12. Murakami Y, Mizuguchi K. Homology-based prediction of interactions between proteins using averaged one-dependence estimators. BMC Bioinform. 2014;15:213.

13. Shen J, Zhang J, Luo X, Zhu W, Yu K, Chen K, et al. Predicting proteinprotein interactions based only on sequences information. Proc Natl Acad Sci U S A. 2007:104:4337-41.

14. You Z, Lei Y, Zhu L, Xia J, Wang B. Prediction of protein-protein interactions from amino acid sequences with ensemble extreme learning machines and principal component analysis. BMC Bioinform. 2013;14(Suppl 8):S10.

15. Zhou H, Jakobsson E. Predicting protein-protein interaction by the mirrortree method: possibilities and limitations. PLoS One. 2013;8:e81100.

16. Zhang QC, Petrey D, Garzón Jl, Deng L, Honig B. PrePPI: a structureinformed database of protein-protein interactions. Nucleic Acids Res. 2013; 41:D828-33.

17. Fukuhara N, Kawabata T. HOMCOS: a server to predict interacting protein pairs and interacting sites by homology modeling of complex structures. Nucleic Acids Res. 2008;36:185-9.

18. Ghoorah AW, Devignes MD, Smaïl-Tabbone M, Ritchie DW. Spatial clustering of protein binding sites for template based protein docking. Bioinformatics. 2011;27:2820-7

19. Chen R, Li L, Weng Z. ZDOCK: an initial-stage protein-docking algorithm Proteins. 2003;52:80-7.

20. Zhang C, Lai L. SDOCK: a global protein-protein docking program using stepwise force-field potentials. J Comput Chem. 2011;32:2598-612.

21. Ritchie DW, Venkatraman V. Ultra-fast FFT protein docking on graphics processors. Bioinformatics. 2010;26:2398-405.

22. Kozakov D, Beglov D, Bohnuud T, Mottarella SE, Xia B, Hall DR, et al. How good is automated protein docking? Proteins. 2013;81:2159-66.

23. Bourgeas R, Basse MJ, Morelli X, Roche P. Atomic analysis of proteinprotein interfaces with known inhibitors: the 2P21 database. PLoS One. 2010;5:e9598.

24. Fuller JC, Burgoyne NJ, Jackson RM. Predicting druggable binding sites at the protein-protein interface. Drug Discov Today. 2009:155-61.

25. Ohue M, Matsuzaki Y, Uchikoga N, Ishida T, Akiyama Y. MEGADOCK: An allto-all protein-protein interaction prediction system using tertiary structure data. Protein Pept Lett. 2014;21:766-78.

26. Mintseris J, Pierce B, Wiehe K, Anderson R, Chen R, Weng Z. Integrating statistical pair potentials into protein complex prediction. Proteins. 2007;69: 511-20.

27. Ohue M, Shimoda T, Suzuki S, Matsuzaki Y, Ishida T, Akiyama Y. MEGADOCK 4.0: an ultra-high-performance protein-protein docking software for heterogeneous supercomputers. Bioinformatics. 2014;30:3281-3.

28. Tuncbag N, Gursoy A, Nussinov R, Keskin O. Predicting protein-protein interactions on a proteome scale by matching evolutionary and structural similarities at interfaces using PRISM. Nat Protoc. 2011;6:1341-54.

29. Mosca R, Céol A, Aloy P. Interactome3D: adding structural details to protein networks. Nat Methods. 2012;10:47-53.

30. Behrens J, Jerchow BA, Würtele M, Grimm J, Asbrand C, Wirtz R, et al. Functional interaction of an axin homolog, conductin, with beta-catenin, APC, and GSK3beta. Science. 1998;280:596-9.

31. Nakamura T, Hamada F, Ishidate T, Anai K, Kawahara K, Toyoshima K, et al. Axin, an inhibitor of the Wnt signalling pathway, interacts with beta-catenin, GSK-3beta and APC and reduces the beta-catenin level. Genes Cells. 1998:3: 395-403.

32. Rose PW, Prlić A, Bi C, Bluhm WF, Christie CH, Dutta S, et al. The RCSB protein data Bank: views of structural biology for basic and applied research and education. Nucleic Acids Res. 2015:43:D345-56.

33. Play Framework. https://www. playframework.com/. Accessed 1 June 2017

34. H2 Database Engine. http://www.h2database.com/html/main.html.. Accessed 1 June 2017

35. MySQL. https://www.mysql.com/. Accessed 1 June 2017.

36. Altschul SF, Madden TL, Schäffer AA, et al. Gapped BLAST and PSI-BLAST: a new generation of protein database search programs. Nucleic Acids Res. 1997;25:3389-402

37. Wasmuth EV, Lima CD. UniProt: the universal protein knowledgebase Nucleic Acids Res. 2016;45:1-12. 
38. Kanehisa M, Furumichi M, Tanabe M, Sato Y, Morishima K. KEGG: new perspectives on genomes, pathways, diseases and drugs. Nucleic Acids Res. 2017;45:D353-61.

39. Cokelaer T, Pultz D, Harder LM, Serra-Musach J, Saez-Rodriguez J. BioServices: a common python package to access biological web services programmatically. Bioinformatics. 2013;29:3241-2.

40. Das J, Yu H. HINT: high-quality protein interactomes and their applications in understanding human disease. BMC Syst Biol. 2012;6:92.

41. Chatr-Aryamontri A, Oughtred R, Boucher L, Rust J, Chang C, Kolas NK, et al. The BioGRID interaction database: 2017 update. Nucleic Acids Res. 2017;45: D369-79.

42. Ohue M, Matsuzaki Y, Ishida T, Akiyama Y. Improvement of the proteinprotein docking prediction by introducing a simple hydrophobic interaction model: an application to interaction pathway analysis. Lecture Notes in Bioinform. 2012:7632:178-87.

43. Ohue M, Matsuzaki Y, Shimoda T, Ishida T, Akiyama Y. Highly precise protein-protein interaction prediction based on consensus between template-based and de novo docking methods. BMC Proc. 2013;7:S6.

44. Matsuzaki Y, Ohue M, Uchikoga N, Akiyama Y. Protein-protein interaction network prediction by using rigid-body docking tools: application to bacterial chemotaxis. Protein Pept Lett. 2014;21(8):790-8.

45. Bekker GJ, Nakamura H, Kinjo AR. Molmil: a molecular viewer for the PDB and beyond. J Cheminform. 2016;8:42.

46. Szklarczyk D, Morris JH, Cook H, Kuhn M, Wyder S, Simonovic M, et al. The STRING database in 2017: quality-controlled protein-protein association networks, made broadly accessible. Nucleic Acids Res. 2017:45:D362-8.

47. Dall'Era MA, Oudes A, Martin DB, Liu AY. HSP27 and HSP70 interact with CD10 in C4-2 prostate cancer cells. Prostate. 2007:67:714-21.

48. Oefner C, Roques BP, Fournie-Zaluski MC, Dale GE. Structural analysis of neprilysin with various specific and potent inhibitors. Acta Crystallogr Sect D Biol Crystallogr. 2004;60:392-6.

49. Allaire M, Li Y, Mackenzie RE, Cygler M. The 3-D structure of a folatedependent dehydrogenase/cyclohydrolase bifunctional enzyme at 1.5 å resolution. Structure. 1998;6:173-82

\section{Submit your next manuscript to BioMed Central and we will help you at every step:}

- We accept pre-submission inquiries

- Our selector tool helps you to find the most relevant journal

- We provide round the clock customer support

- Convenient online submission

- Thorough peer review

- Inclusion in PubMed and all major indexing services

- Maximum visibility for your research

Submit your manuscript at www.biomedcentral.com/submit

) Biomed Central 